\title{
"An Approach for Intersection Prevention in Transmission Channel Using Correlation Matrix and Tree Construction Model in V2x Framework"
}

GraceShalini T ( $\sim$ graceshaliniphd@gmail.com )

Velammal College of Engineering and Technology https://orcid.org/0000-0002-0016-3702 Jenicka S

Sethu Institute of Technology

\section{Research Article}

Keywords: vehicular ad hoc networks, intersection, forwarding zone computation, 55 correlation matrix based intersection prevention, tree construction, availability of channel

Posted Date: March 17th, 2021

DOI: https://doi.org/10.21203/rs.3.rs-306871/v1

License: (c) (i) This work is licensed under a Creative Commons Attribution 4.0 International License.

Read Full License 



\begin{abstract}
The delivery of information in vehicular ad hoc networks (VANETs) is regarded as demanding because of high mobility and invariable topological difference. One major concern in VANET framework is the occurrence of simulated intersection at which the packet reception probability becomes complicated for prediction and the model turns out to be highly complex. So as to overcome this dispute, a new framework is introduced which in turn prevents the intersection among the transmission path. Initially, the system model is initialized and the availability of channel is estimated. Based on the strength and size of data to be transmitted, the availability of channel is computed. After that, the best forwarding zone computation is made for minimizing the redundant data packets flow. To check the priority of packet and occurrence of packet collision, tree construction based data strength transmission is employed. Finally, the prevention of intersection or collision between the transmission channels is checked by means of correlation matrix based intersection prevention approach by monitoring the neighbor node. Then the data packets were forwarded in an efficient manner without any intersection between the frames. The performance analysis is estimated in terms of network lifetime, packet delivery ratio, packet collision; inter node collision, throughput, and end-to-end delay.
\end{abstract}

Index Terms - vehicular ad hoc networks, intersection, forwarding zone computation, correlation matrix based intersection prevention, tree construction, availability of channel.

\section{INTRODUCTION}

In VANET the demand of immense traffic rate leads to the continuous growth of bandwidth demand. The service of VANET becomes highly advanced for the enhancement of traffic efficiency and road safety improvement on targeting a highly comfortable driving and the experience of travelling. For addressing such a requirement of capacity, it is essential to ensure the exploitation of optimal radio resources that were available. The direction and speed of the necessitate vehicles need to alter their distance and position recurrently. As a result, the vehicles have to keep on altering their point of communication at the time of vehicle information transmission mechanism. All through handover or handoff, vehicles block their receiving services from their communication points that were connected previously and connect with another for the receiving services [1]. VANETs consist of some special features that involve road pattern restrictions, large scale network sizes, self-organization, high mobility, no constraints of energy, etc. [2]. The vehicular networks is considered as a challenging technology which provides smart vehicles for exchanging wireless information between them so as to attain excess safer and convenient system of transportation. This information might comprise data regarding the conditions of traffic, adaptive assistance of trip, alarms and warnings, and availability of parking gas station with infotainment applications. 
VANET is regarded as the extended mobile ad-hoc networks application that finds out new routes to the points of communication during handoff time. The communicating vehicle prompts re-routing for the discovery of new communication points positioned further away or nearby [3]. The procedure of Handoff provided for VANET should ensure flawless performance on behalf of supporting these applications. Because of vehicle's direction and unpredictable velocity, in VANETs handoff is a challenging task [4, 5]. The application of VANET requires the moving vehicle's position to assist specific user services. In VANET, the localization of conventional global

positioning system (GPS) does not assure the position based application due to availability concern, signal errors, localization proximity, communication management, and handoff. These are the incorporated blocks and disputes for the management of mobility $(\mathrm{MM})$ in VANETs $[6,7]$.

In the scenario of urban VANET, usually vehicles are not dispersed consistently in the region of the City road. For these, the network might be fragmented and the proficient communication among V2X get affected [8,

9]. In addition, the impediments existence like other constructions or buildings could be the interruption basis to radio signal, which in turn leads to

communication failure, as they are in the communication zone of each other. Most of the routing protocols proposed intended for Urban VANETs do not allow the probable hindrance with a negative routing impact, which in turn designates that the data flow direction system towards the destination in view of the obstacles existence was a significant research concern.

One more significant aspect is a forwarding zone selection in that way. After that, the possible selection of forwarding node on the forwarding zone is one more challenge for the efficient data transmission.

The remaining part of the paper is organized as follows: Section 2 offers various existing mechanisms presented so far. Section 3 provides the proposed algorithm and performance estimation were provided in section 4 . Section 5 offers the concluding statement.

\section{RELATED WORK}

This section is the description of various existing mechanism employed so far.
A system is presented [4] for Adaptive intersection selection using Ant colony Optimization approach, a problem of finding a challenging route concern for the multiple QoS constraint. In addition, the outcomes in the urban framework reveals that the AISM outperforms the traditional protocols by extensive simulation environment in terms of Packet delivery ratio, hop count, and average delay [10]. An adaptive multichannel medium access control (MAC) high-throughput protocol, specifically, AHT-MAC [11] was proposed that could manage the transmission of data over SCHs. By means of this AHT-MAC, range of data broadcasting was attuned in accordance with the range of beacon transmission over $\mathrm{CCH}$ in order that a transmitting node might establish suitable candidate's communication and arrange resources available for both communication nodes prior to transmission.

A scheme of link quality-dependent dissemination of security message on behalf of urban VANETs was presented [12]. A wide-ranging connectivity of physical channel computation technique was suggested for estimating the probability of connectivity among vehicles precisely. A score-dependent mechanism of priority allocation was suggested for candidate forwarders (CFs) so as to manage the controversy between $\mathrm{CFs}$.

The authors [13] utilized the controller SDN for mitigating the congestion of Vehicle-to-Vehicle communications even as routing information on the segments of road. This was attained by competent VANET bandwidth utilization on the road segments. In contradiction of current assistance, the suggested SDN controller offers a new mechanism of routing which considers the other traditional routing paths that were relaying information in VANET already. A new routing request was found out so that there is no road segment which gets congested through the multiple crossing routing paths.

Intermittent hello packets was suggested [14] for the establishment of neighboring list that furthermore employ information regarding the neighbors, with the dynamic neighbor distance and neighbors direction, for the selection of forwarding node. A hybrid of neighbor and distance list forwarding broadcast protocol was considered. This article in turn mainly focuses on decreasing the delay of broadcast and enhancing the range of dissemination in VANET thereby avoiding the impacts of changing the topology of network. The road 
vehicles density was a significant aspect for the application of time-critical security.

The authors [15] suggested an approach of Multi-metric Power Control (MPC), that employs channel states and application requirements for determining a broadcast power intended for safety communication. The MPC offers a best-effort scheme for satisfying the range of coverage necessity of a message as signified through the application. Additionally, the perception differentiated among types of message for providing coverage discrimination.

The authors [16] considered a design of physical (PHY)/MAC cross-layer depending on transmit power adaptation (TPA) and transmit antenna selection (TAS). A spatial multiplexing zero-forcing Bell-labs layered space-time (ZF-VBLAST) was considered over timevarying multiple-input and multiple-output (MIMO) flat fading channel that were to be implemented in the vehicle-to-vehicle $(\mathrm{V} 2 \mathrm{~V})$ communication.

A Trunk Road dependent Protocol of Geographic Routing in Urban VANETs (TRGR) [17] was presented. This protocol in turn aims at resolving the data acquisition problem in the traditional system of trunk coordinated control mechanism. On considering the real physical distinctiveness of trunk lines, it formulates the entire utilization of trunk lines traffic flow and the neighboring road network, offers a transmission of realtime data routing design, and provides a vehicle network routing protocol in this particular form.

The authors [18] suggested a WAVE acquiescent improvement to the existing protocol IEEE 802.11p that targets the delivery of prioritized safety information whereas provisioning the non-safety messages dissemination at the same time. The suggested

technique relies on the generation of dynamic beacons for mitigating the congestion of channel and incompetent utilization of bandwidth through reducing beacons transmission frequency.

An effective strategy of charging information transmission (ECTS) [19] on behalf of spatio-temporal coordinated services of vehicle-to-vehicle (V2V) charging was considered. In particular, depending on the mobile edge computing (MEC) concepts and hybrid vehicular ad hoc networks, a scalable and effective framework of communication was designed initially to shrink the costs of communication.
The authors [20] highlighted the major intention of introducing an optimal route path process for minimizing the link failure chances and energy consumption reduction of nodes in the network. In this article, an algorithm of the modified route optimal path at which the nodes were in cluster form was projected in the direction of achieving this objective. In this, the scheme of mobility prediction was employed for the stability of the network, and the two-tier method was employed in the minimization of energy consumed in the course of a Location Aided Routing (LAR) protocol. The behavior of network [21] was analyzed in a sensible area of simulated intersection wherever the packet reception probability become complicated for predicting and the model turn out to be complex highly. In that situation, a critical analysis was presented on the current US and EU decentralized congestion control protocols performance whereas their performance was estimated regarding the accuracies of tracking needed by the application of Intelligent Transportation System (ITS).

\section{PROPOSED WORK}

This section depicts the proposed adaptive intersection based channel selection strategy in detail. The flow of the proposed technique is shown below:

\section{A. Node deployment}

Let us envisage a VANET environment that consists of vehicles, which is a typical scenario in urban areas. The initialization of system model is made at first by considering the number of transmitters, number of receivers, number of packets, packet size, number of frames, frame size, data strength and number of iterations based on the vehicle node. The number of iterations can be varied as per the number of vehicles considered. The packet collision for each vehicle is being initialized. It was assumed that there was $\mathrm{N}$ number of nodes that were moving at some distance as per the reference region model of group mobility. The entire nodes in the model have an equivalent range of transmission. Each node is capable of transferring information to the neighboring nodes. 
B. Channel availability and best forwarding zone prediction:

After the initialization of parameters, the request for all available channels is made in case the user is ready to transmit data. Based on the strength and size of data to be transmitted, the availability of channel is computed. The channel availability is offered based on the priority
Probability estimated based on the strength of data

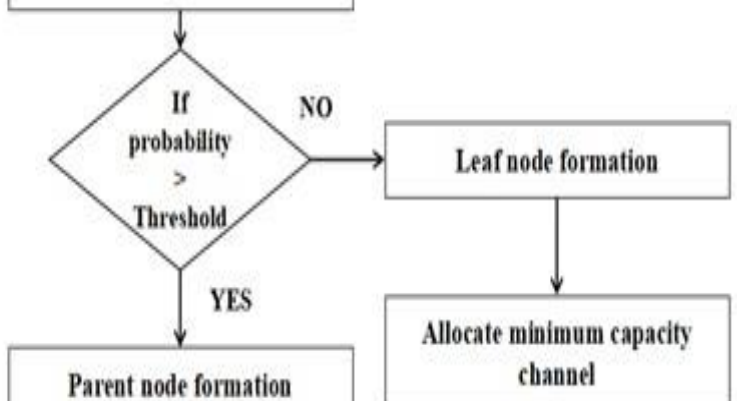

Apply channel detection Technique

Channel Availability Prediction

Obtain channel feedback from Neigbour cell

Computed best forwarding zone

Allocate maximum capacity channel

Figure 1 Flow of proposed strategy

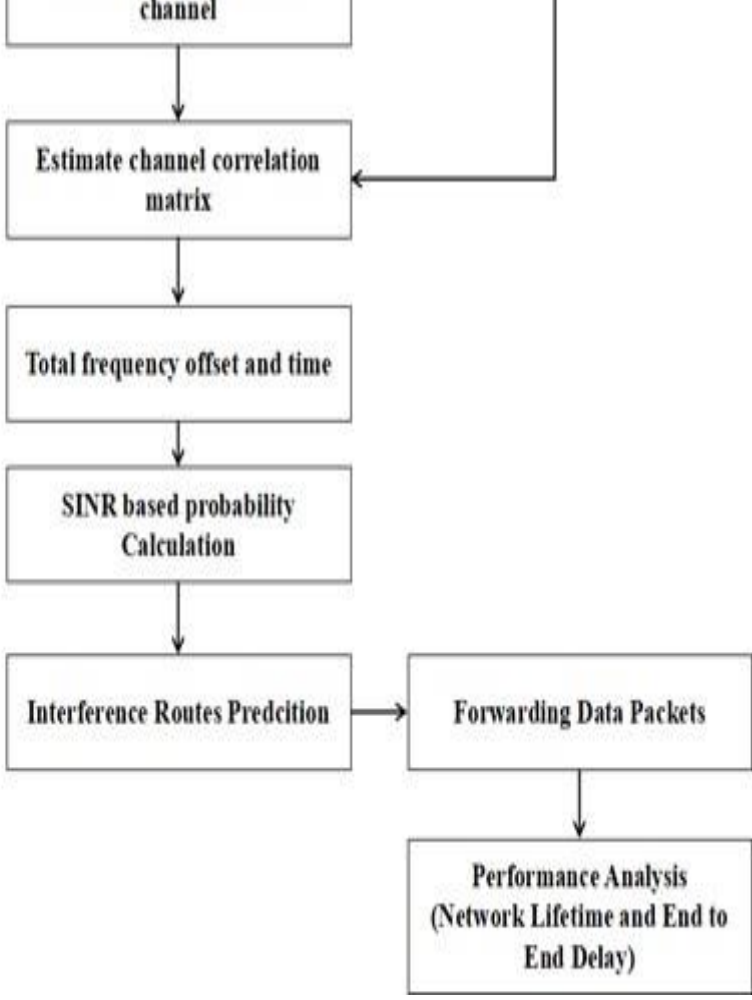

of data packets or messages that were to be transmitted. Users are transmitting either or in handoff mode on various timestamps. Depending on the arrival of user, subcarrier should assign appropriate channel or frame for users. Then, the computation of best forwarding zone is made. So as to minimize the redundant data packets flow, the forwarding zone is being set so that 
the entire nodes present in the zone were in the wireless range of one another. For achieving these criteria, the forwarding zone with sector shape having best angle of $60 \circ$ towards the destination is being set. This fixed

forwarding angle in turn limits the maximum distance in the forwarding zone. This in turn will allow the nodes present in forwarding zone to heed each other's transmission of packet, in that way allowing only one node to communicate the data packet in the forwarding zone.

\section{Tree construction based on strength of transmission data:}

In this the packets are prioritized by constructing the key that is based on number of user, route node, shift center point of packet, packet size, bandwidth of

packet, and number of packets received. This is done based on each transmitter and receiver tree, and their channel availability. This key construction is made by means of the novel approach termed tree construction based on strength of transmission of data in order to check the priority of packet and occurrence of packet collision. This tree construction mainly depends on the strength of user and packet size. For each node and available channel, the lambda is increased in each iteration. The algorithm for this tree construction based transmission of data is shown below:

Algorithm 1: Tree based data strength Priori

Input : Frame packet from each vehicle $V_{P_{F}}$ (data,

packet priority DP)

Output: formed data Priority tree

$P T_{\text {high }}, P T_{\text {medium }}, P T_{\text {low }}, P T_{\text {normal }}$

Step 1: read the input $V_{P_{F}}$ from the vehicle node with header information.

Step 2: Check the header and separate data packet based on weight value from vehicle.

Step 3: Construct the priority table in tree formation,

$$
\begin{aligned}
& \text { if DP need } \\
& \qquad \begin{aligned}
& \text { if } V_{P_{F}}==P T_{h i g h} \text { then } \\
& \text { Add the packet to root node of the tree }
\end{aligned}
\end{aligned}
$$

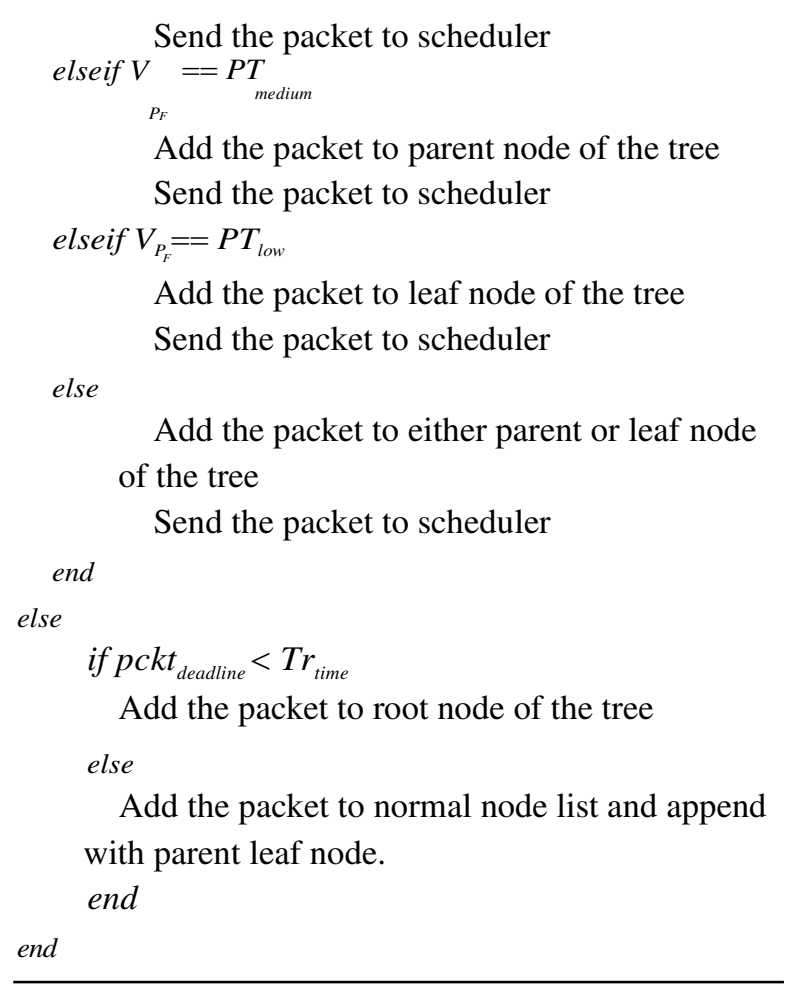

In the tree construction based data strength priori, the input is the frame packet from each vehicle. Initially, the information from the vehicle is read from the header information. By checking the header, data packet is separated based on vehicle's weight value. In the form of tree, the priority tree is constructed. The packet with high priority is added to the root node of the tree by sending packet to the scheduler. Similarly, the packet of medium priority is added to the leaf node of tree by sending packet to the scheduler. Likewise, those packets with low priority is added to either parent or leaf node of the tree by sending the packet to scheduler. If the deadline of packet is less than transmission time, the packet is added to root node of the tree or else the packet is added to normal node list and is appended with the parent leaf node.

\section{Prevention of Intersection routes}

During this data transmission process, there will be some occurrence of intersection. So as to evade this type of intersection between the channel and data transmission, the concept of intersection routes prevention by monitoring neighbor nodes was introduced. In this, the initialization of vehicle correlated matrix and vehicle user gain is made. Then for each correlated matrix of the vehicle, their minimum Eigen vector of data strength is estimated. To 
achieve SINR threshold, the vehicle 1 with N1 channel must satisfy the probability condition. The noise power spectral density is computed which in turn describes the intersection occurred among the vehicles. The Eigen vector estimation step is carried till the fixed base station is attained for the transmission of data. These steps were carried similarly for vehicle 2 .

In the prevention of intersection routes, correlation matrix formation is introduced in which the probability, data strength, available gain of the path, availability of base station is considered. The request from the neighbor or another frame causes intersection which leads to formation of another route. So as to prevent this, the intersection prevention approach is employed.

The power spectral probability is estimated AND by increasing the number of node, the collision occurrence is checked and prevented.

Algorithm 2: Prevention of intersection of Nodes:

1. Initialize vehicle correlated matrix $C_{1}$ and $C_{2}$ and

vehicle user gains, ${ }^{1}$ and ${ }^{2}$.

$2_{n}^{2}$, For where $n=1,2, \ldots, N_{1}$, its a minimum eigen vector of 1 data strength. achieve its SINR threshold, the following probability in should be satisfied, $\frac{P_{v_{i}}{ }^{n}}{N+\sum_{i=1}^{W_{i}} P_{V_{i}}^{n} G_{V_{i}}^{n}} \geq \gamma$

Here, $N_{0}$ is the noise power spectral density, $\sum_{i=1}^{K} P_{V_{i}}{ }^{n} G_{V_{i}}{ }^{n}$ describes the total interference of the vehicles who utilize the channel $N_{1}, \gamma$ is the SINR threshold. $K$ indicates the all vehicle which are available in network. 4. Repeat step 2 until a fixed base station obtained for data transmission.

5. For each correlated matrix of vehicle 2 $C_{n}^{2}$, where $n=1,2, \ldots, N$, it's a minimum Eigen vector of data strength.

6. For the vehicle 2 who uses channel $\quad N_{2}$, to achieve its SINR threshold, the following probability in should be satisfied,

$$
\frac{P_{V_{2}}^{n}}{N+\sum_{i=1}^{\mathrm{K}_{V_{i}}} P_{V_{i}}^{n} G^{n}} \geq \gamma
$$

Here, ${ }{ }_{0}$ is the noise power spectral density, $\quad \sum_{i=1}^{K} R_{i} G_{V_{i}}{ }^{n}$ describes the total interference of the vehicles who utilize the channel $N_{2} . \gamma$ is the SINR threshold. $K$ indicates the all vehicle which are available in network. 7. Repeat step 4 until a fixed base station obtained for data transmission.

Thus, the intersection of nodes will be prevented by monitoring the neighbor nodes. At last, the data packets were forwarded in an efficient manner. At last, the performance analysis is estimated in terms of network lifetime and end-to-end delay.

\section{Performance ANAlysis}

The performance analysis of the proposed system is estimated and the outcomes are provided in this section. The comparative analysis is made with existing techniques [22, 23]. The computation of performance was made in terms of network lifetime and delay.

3. For the vehicle 1 who uses channel $N_{1}$, to

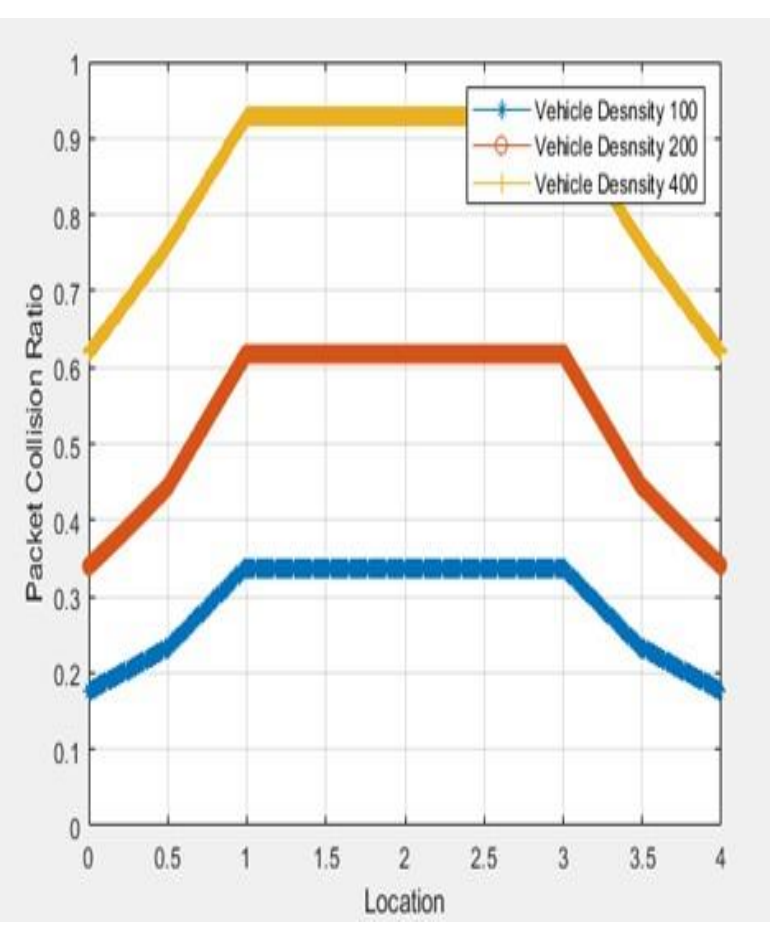

Figure 2 performance analysis of packet collision ratio

The performance analysis of packet collision ratio is signified in figure 2 . The packet collision ratio for 
vehicle density 100,200 , and 400 is estimated with respect to location.

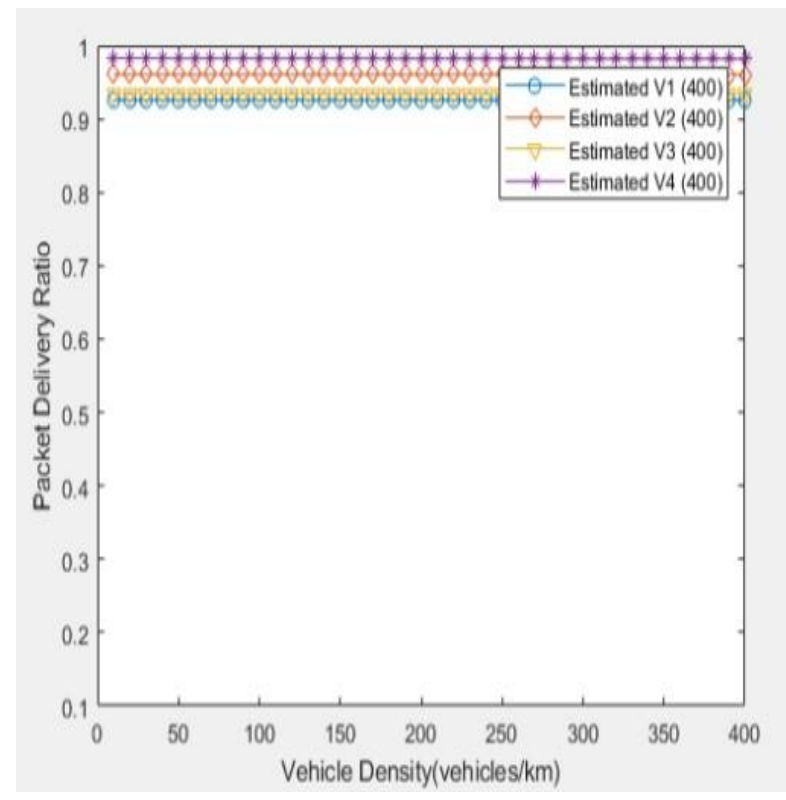

Figure 3 performance analysis of packet delivery ratio

PDR is referred to as the amount of traffic amount that gets at the destination effectively similar to the fraction of generated traffic by the source node. Figure 3 depicts that the packet delivery ratios of estimated V1, V2, V3, and V4 with respect to the vehicle density.

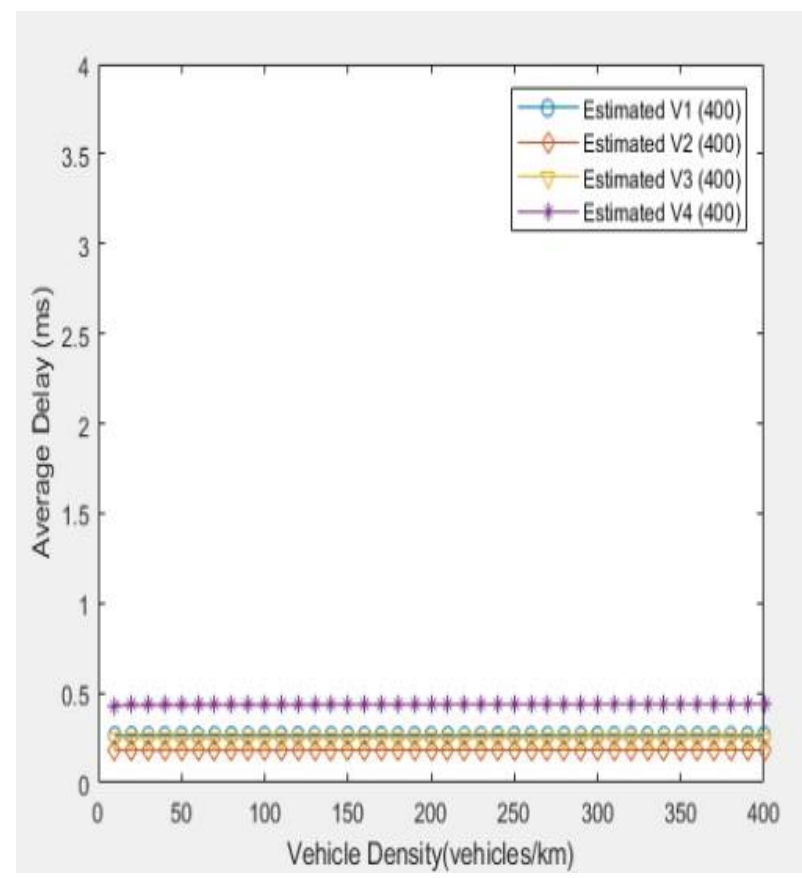

Figure 4 performance analysis of Average delay (ms)

Average E2E delay signifies the time difference among the packet reception through the target and the instant the source created it; this covers all delays possible encountered through a packet. Figure 4 depicts that the Average E2E delay of estimated V1, V2, V3, and V4 with respect to the vehicle density.

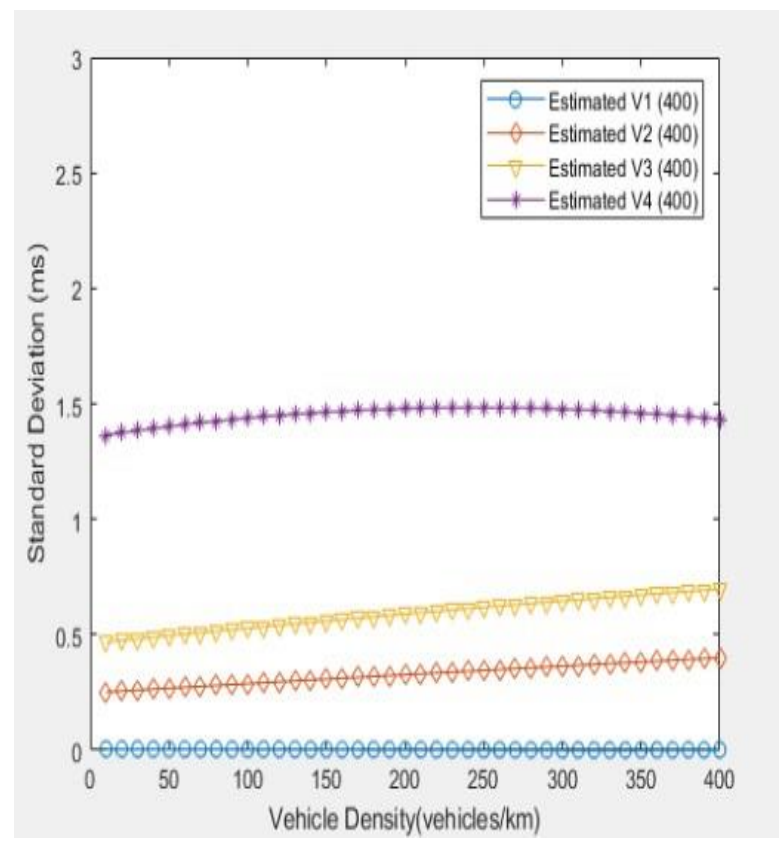

Figure 5 performance analysis of standard deviation Vs vehicle density

Figure 5 is the representation of performance analysis of standard deviation Vs vehicle density. The performance analysis is carried out in terms of estimated vehicles V1, V2, V3, and V4. The standard deviation increases with respect to estimated vehicles increment. 


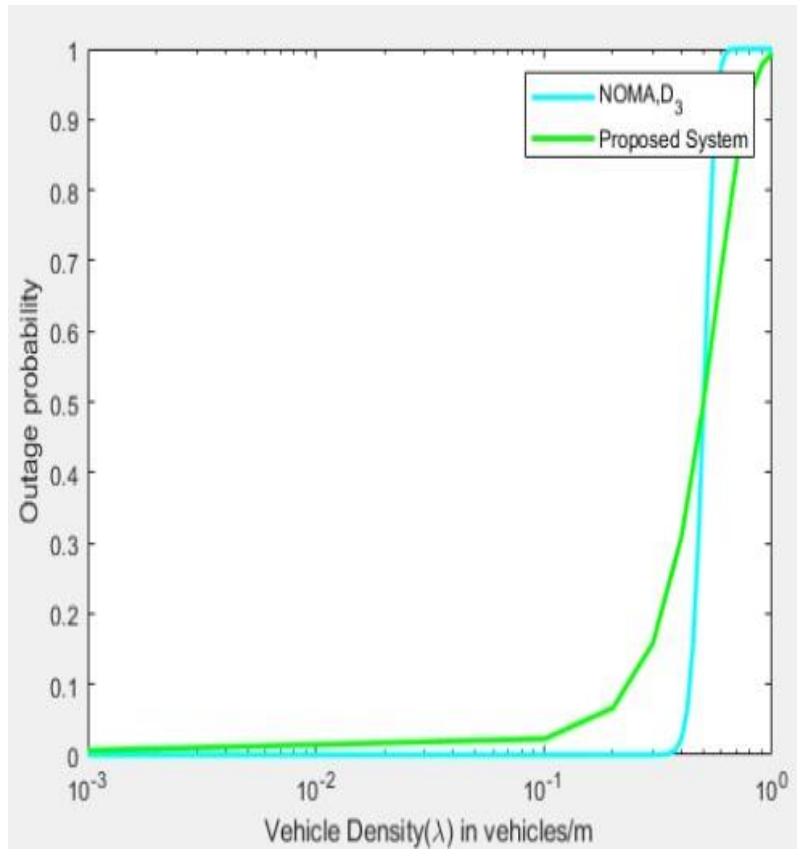

Figure 6 performance analysis of outage probability vs vehicle density

The outage probability in terms of vehicle density is depicted in figure 6 . The comparative analysis is carried out between the proposed technique and $\mathrm{NOMAD}_{3}$ which shows that the proposed mechanism is having better outage probability which increases gradually.

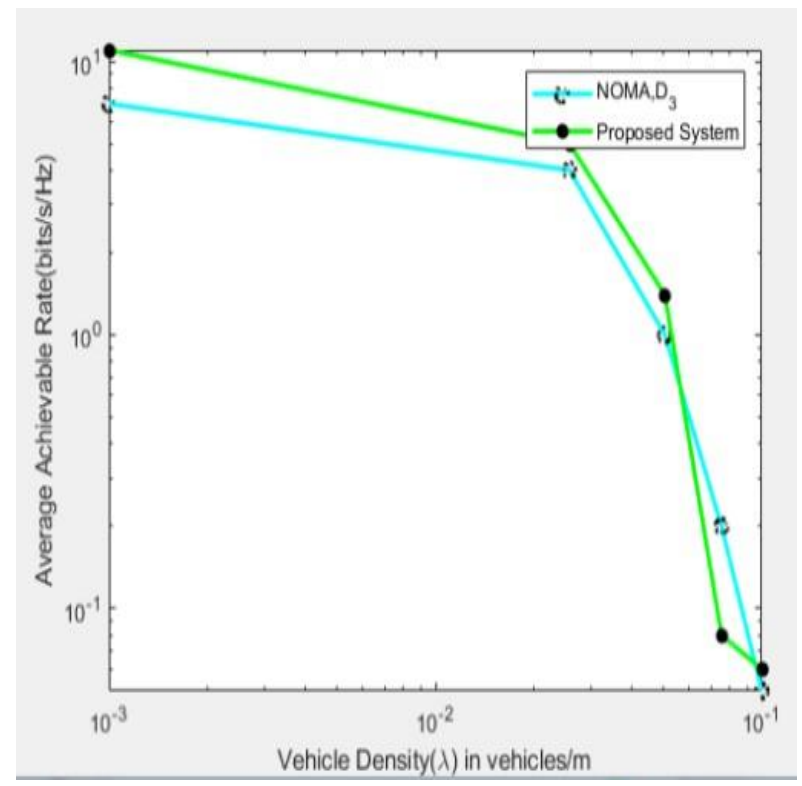

Figure 7 performance analysis of Average achievable rate
The average achievable rate in terms of vehicle density is represented in figure 7 . The comparative analysis is carried out between the proposed technique and $\mathrm{NOMAD}_{3}$ which shows that the proposed mechanism is having better achievable rate in an average and in turn decreases slowly.

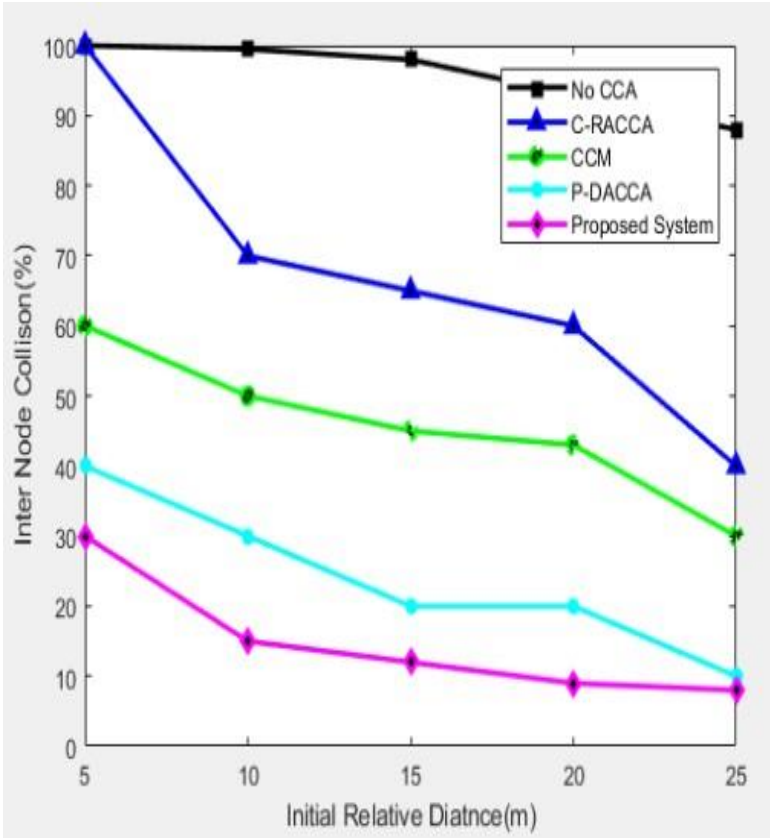

Figure 8 comparative analysis of Inter node collision vs initial relative distance

Figure 8 is the representations of comparative analysis of inter node collision vs. initial relative distance (m). The analysis is carried out between the existing techniques like NoCCA, C-RACCA, CCM, P-DACCA and the proposed methodology. The inter node collision is low for proposed system on comparing other methodologies. 


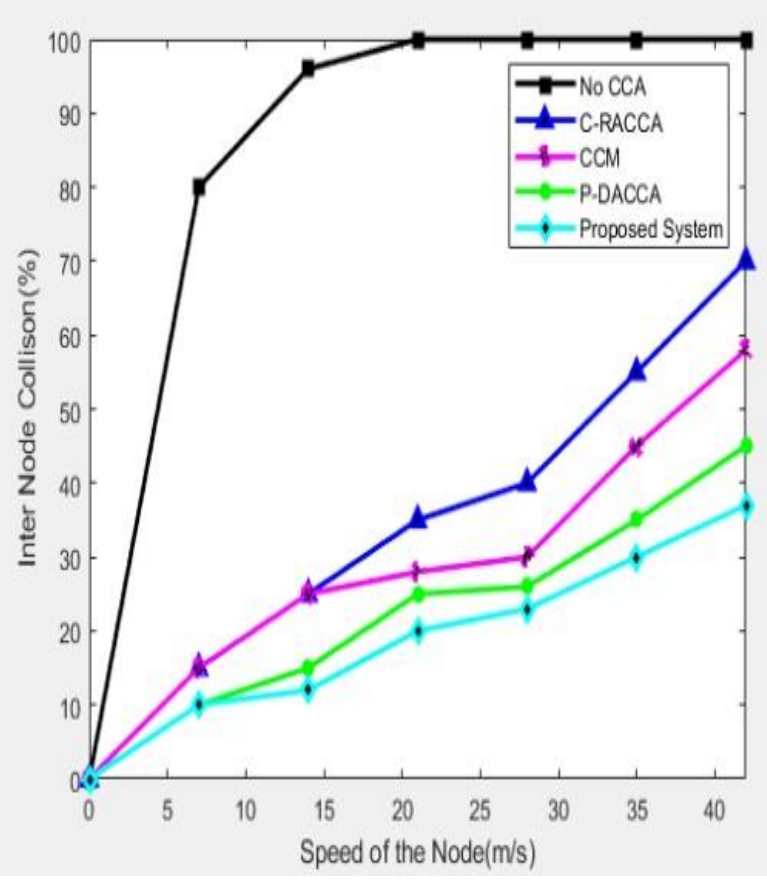

Figure 9 comparative analysis of Inter node collision Vs speed of the node

Figure 9 is the depictions of comparative analysis of inter node collision Vs. node speed $(\mathrm{m} / \mathrm{s})$. The comparison was carried out between the existing techniques like NoCCA, C-RACCA, CCM, P-DACCA and the proposed methodology. The inter node collision is low for proposed system on comparing other methodologies. As the speed of node increases, the inter node collision increases gradually.

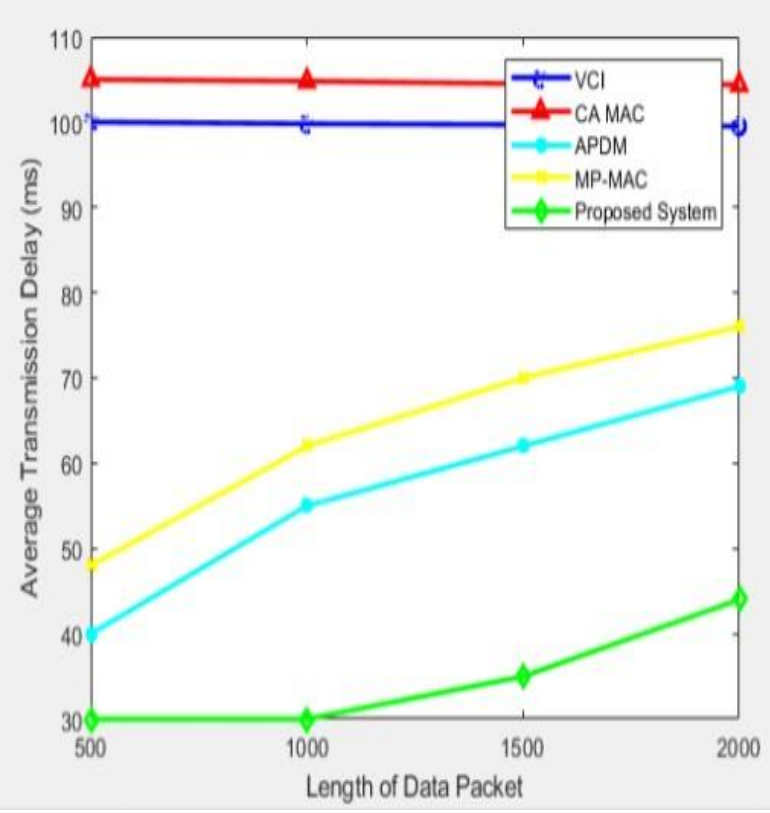

Figure 10 comparative analysis of Average transmission delay (ms)

The average transmission delay in terms of data packet length is represented in figure 10. The comparative analysis is carried out between the proposed technique and existing techniques like VCI, CA MAC, APDM, and MP-MAC which shows that the proposed mechanism is having lower transmission delay in an average which increases with the length of data packet.

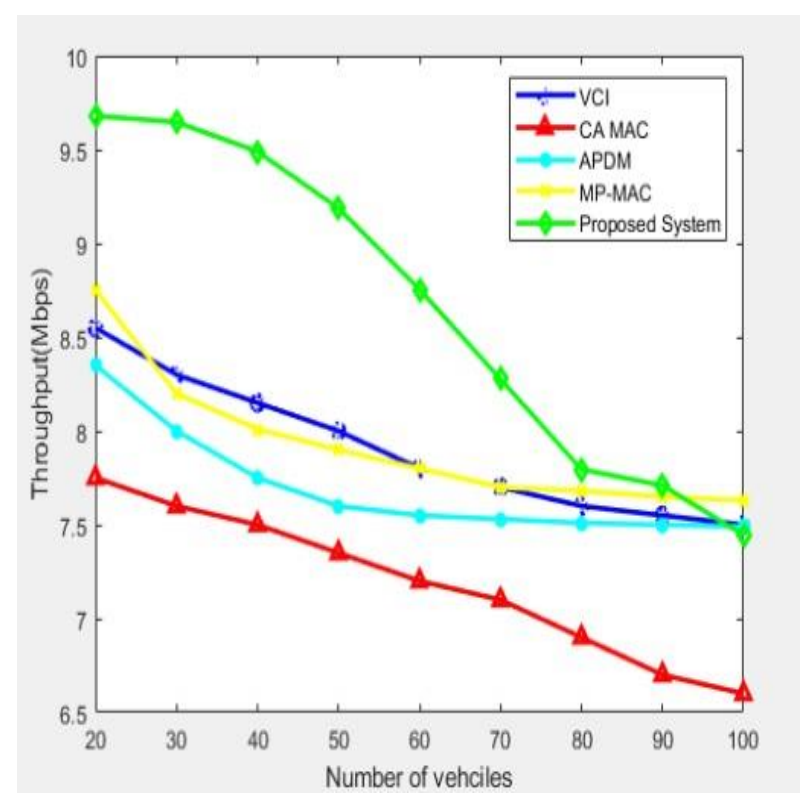

Figure 11 Throughput analysis

The comparative analysis of throughput in terms of number of vehicles is represented in figure 11. The comparative analysis is carried out between the proposed technique and existing techniques like VCI, CA MAC, APDM, and MP-MAC which shows that the proposed mechanism is having better throughput performance which decreases with increase in number of vehicles.

\section{CONCLUSION}

An efficient technique was introduced for the mitigation of intersection occurrence in the channel availability, which is an attempt towards offering a safe transmission environment. This $\mathrm{V} 2 \mathrm{~V}$ approach addresses internodes collision avoidance. The computation of best forwarding zone was made for minimizing the redundant data packets flow. In order to check the packet priority and packet collision, tree 
construction based data strength transmission was introduced. Then, the prevention of intersection or collision between the transmissions channels was checked by correlation matrix based intersection prevention technique on monitoring the neighbor node. After that, the data packets were forwarded without any intersection in an efficient manner between the frames. The performance analysis was made and comparisons were carried with existing techniques in terms of network lifetime, packet delivery ratio, packet collision, inter node collision, end-to-end delay, and throughput.

\section{REFERENCES}

[1] R. Dutta and R. Thalore, "A Review of Various Routing Protocols in VANET," International Journal of Advanced Engineering Research and Science, vol. 4, 2017.

[2] T. Baker, J. M. García-Campos, D. G. Reina, S. Toral, H. Tawfik, D. Al-Jumeily, et al., "Greeaodv: an energy efficient routing protocol for vehicular ad hoc networks," in

International Conference on Intelligent Computing, 2018, pp. 670-681.

[3] C. Ghorai and I. Banerjee, "A robust forwarding node selection mechanism for efficient communication in urban VANETs," Vehicular communications, vol. 14, pp. 109121, 2018.

[4] A. Srivastava, A. Prakash, and R. Tripathi, "An adaptive intersection selection mechanism using ant Colony optimization for efficient data dissemination in urban VANET," Peerto-Peer Networking and Applications, pp. 119, 2020.

[5] A. A. Taleb, "VANET Routing Protocols and Architectures: An Overview," JCS, vol. 14, pp. 423-434, 2018.

[6] S. More and U. Naik, "Novel Technique in Multihop Environment for Efficient Emergency Message Dissemination and Lossless Video Transmission in VANETS," Journal of Communications and Information Networks, vol. 3, pp. 101-111, 2018.

[7] R. Purkait and S. Tripathi, "Fuzzy Logic Based Multi-criteria Intelligent Forward Routing in VANET," Wireless Personal Communications, pp. 1-27, 2019.
[8] L. Liu, C. Chen, B. Wang, Y. Zhou, and Q. Pei, "An efficient and reliable QoF routing for urban VANETs with backbone nodes," IEEE Access, vol. 7, pp. 38273-38286, 2019.

[9] W. Liu, X. He, Z. Huang, and Y. Ji, "Transmission Capacity Characterization in VANETs with Enhanced Distributed Channel Access," Electronics, vol. 8, p. 340, 2019.

[10] B. E. Y. Belmekki, A. Hamza, and B. Escrig, "Performance analysis of cooperative NOMA at intersections for vehicular communications in the presence of interference," Ad Hoc Networks, vol. 98, p. 102036, 2020.

[11] Y. Cao, H. Zhang, Y. Fang, and D. Yuan, "An Adaptive High-Throughput Multi-Channel MAC Protocol for VANETs," IEEE Internet of Things Journal, 2020.

[12] X. Zhang, Q. Miao, and Y. Li, "An Adaptive Link Quality-Based Safety Message Dissemination Scheme for Urban VANETs," IEEE Communications Letters, vol. 22, pp. 2104-2107, 2018.

[13] M. S. Rayeni and A. Hafid, "Routing in heterogeneous vehicular networks using an adapted software defined networking approach," in 2018 Fifth International Conference on Software Defined Systems (SDS), 2018, pp. 25-31.

[14] Q. Huang and F. Liu, "An Efficient Adaptive Broadcast Protocol for Different Scenarios in VANETs," Recent Patents on Computer Science, vol. 10, pp. 131-139, 2017.

[15] S. A. A. Shah, E. Ahmed, F. Xia, A. Karim, M. A. Qureshi, I. Ali, et al., "Coverage differentiation based adaptive tx-power for congestion and awareness control in vanets," Mobile Networks and Applications, vol. 23, pp. 1194-1205, 2018.

[16] A. Triwinarko, I. Dayoub, M. ZwingelsteinColin, M. Gharbi, and B. Bouraoui, "A PHY/MAC cross-layer design with transmit antenna selection and power adaptation for receiver blocking problem in dense VANETs," Vehicular Communications, vol. 24, p. $100233,2020$.

[17] D. Wu, H. Li, X. Li, and J. Zhang, "A Geographic Routing Protocol Based on Trunk Line in VANETs," in Cyberspace Data and 
Intelligence, and Cyber-Living, Syndrome, and Health, ed: Springer, 2019, pp. 21-37.

[18] N. Gupta, A. Prakash, and R. Tripathi,

"Adaptive beaconing in mobility aware clustering based MAC protocol for safety message dissemination in VANET," Wireless Communications and Mobile Computing, vol. 2017, 2017.

[19] G. Li, C. Gong, L. Zhao, J. Wu, and L.

Boukhatem, "An Efficient Reinforcement Learning based Charging Data Delivery Scheme in VANET-Enhanced Smart Grid," in 2020 IEEE International Conference on Big Data and Smart Computing (BigComp), 2020, pp. 263-270.

[20] U. Agballa, A. Obiniyi, and B. Ayeni, "Design of an Improved Energy Efficient Routing Protocol in VANET using a Modified RouteOptimal Path Algorithm," reason, vol. 12, 2019.

[21] C. Iza-Paredes, A. M. Mezher, M. Aguilar Igartua, and J. Forné, "Game-theoretical design of an adaptive distributed dissemination protocol for VANETs," Sensors, vol. 18, p. 294, 2018.

[22] S. Haider, G. Abbas, Z. H. Abbas, S. Boudjit, and Z. Halim, "P-DACCA: A probabilistic direction-aware cooperative collision avoidance scheme for VANETs," Future Generation Computer Systems, vol. 103, pp. 117, 2020.

[23] V. Nguyen, T. T. Khanh, X. Q. Pham, G. W. Lee, and E. N. Huh, "Performance analysis of adaptive MAC protocol in VANETs considering the potential impact on throughput and transmission delays," International Journal of Communication Systems, vol. 33, p. e4172, 2020. 


\section{Figures}

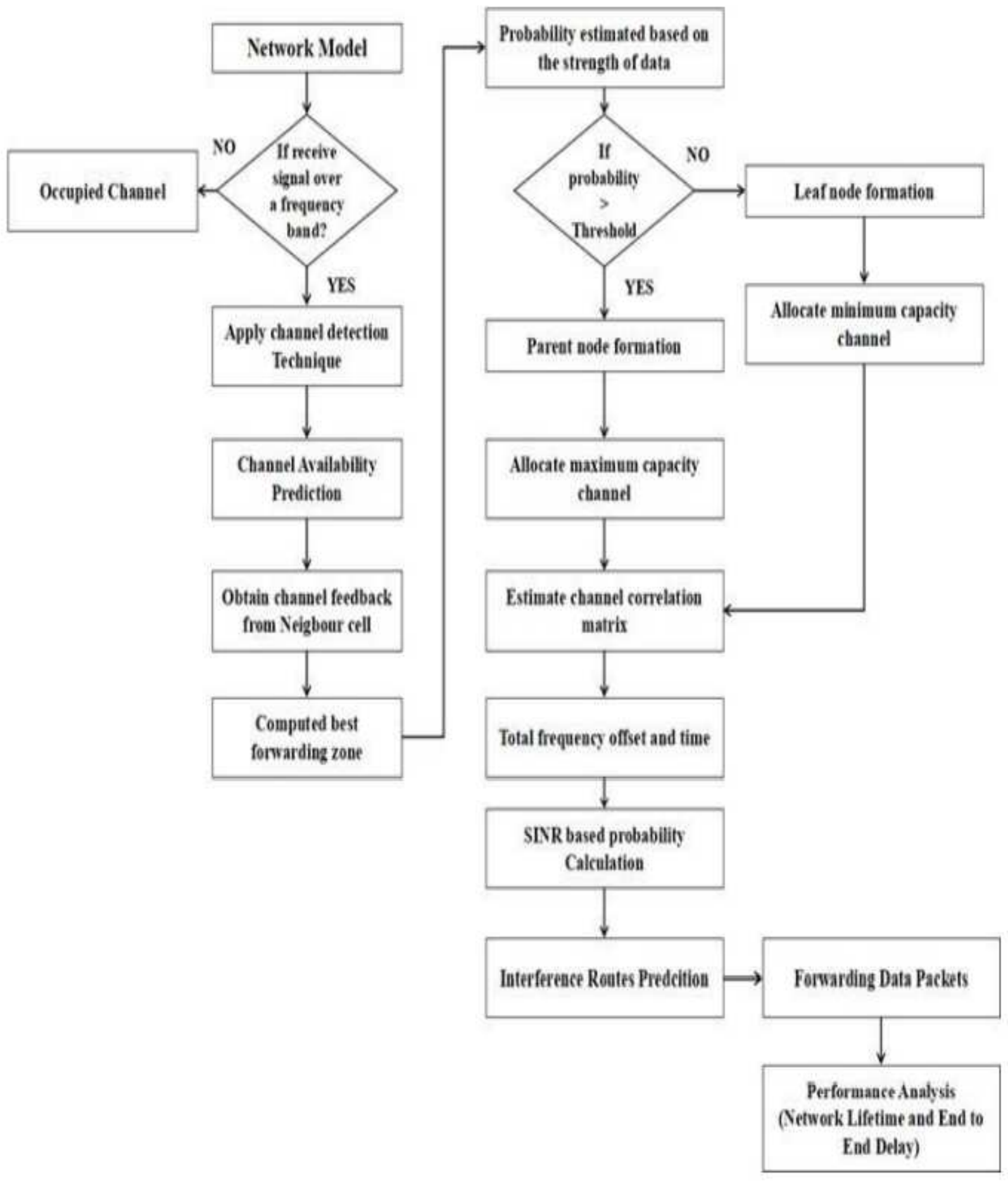

Figure 1

Flow of proposed strategy 


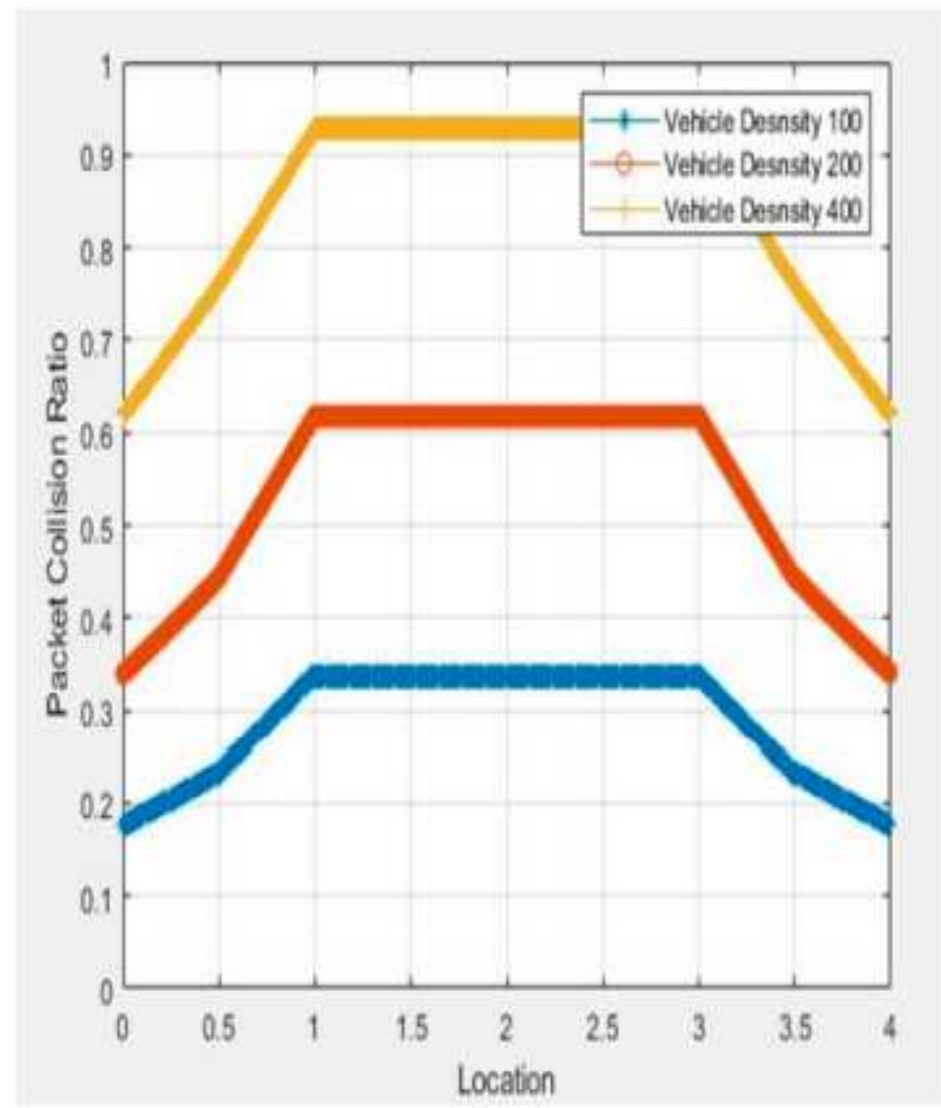

Figure 2

performance analysis of packet collision ratio

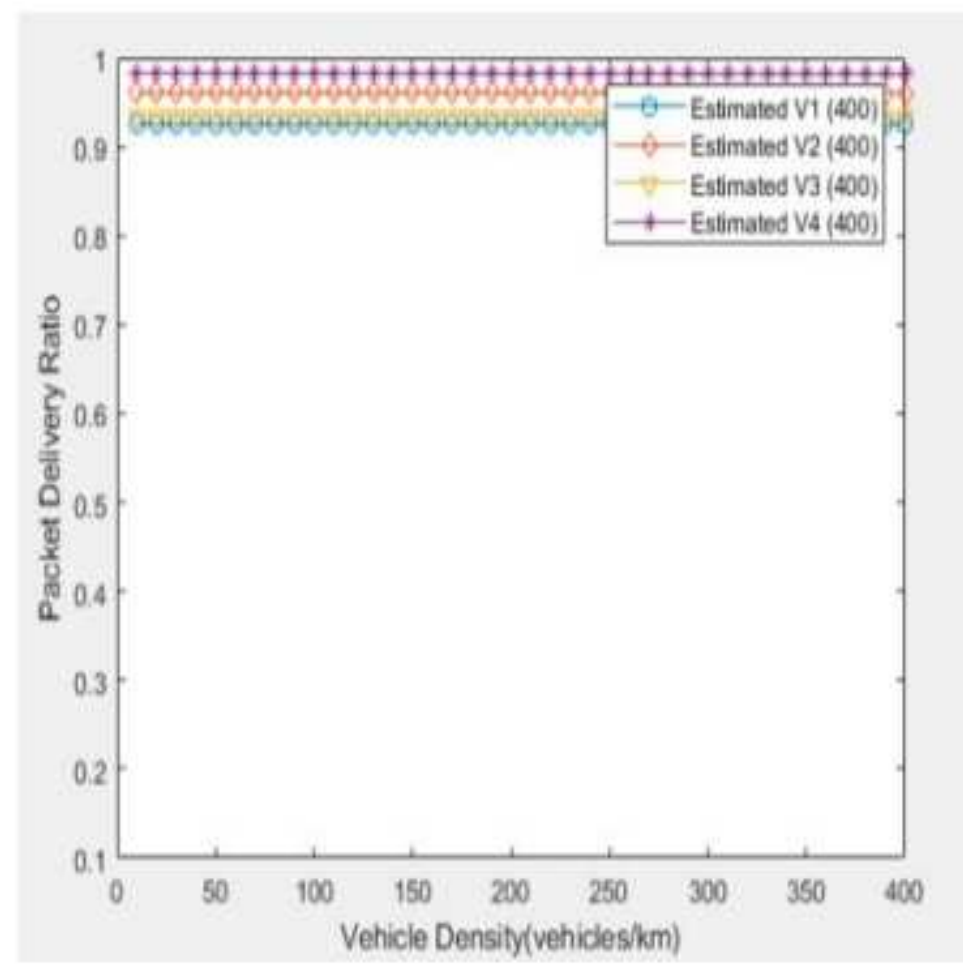

Figure 3 
performance analysis of packet delivery ratio

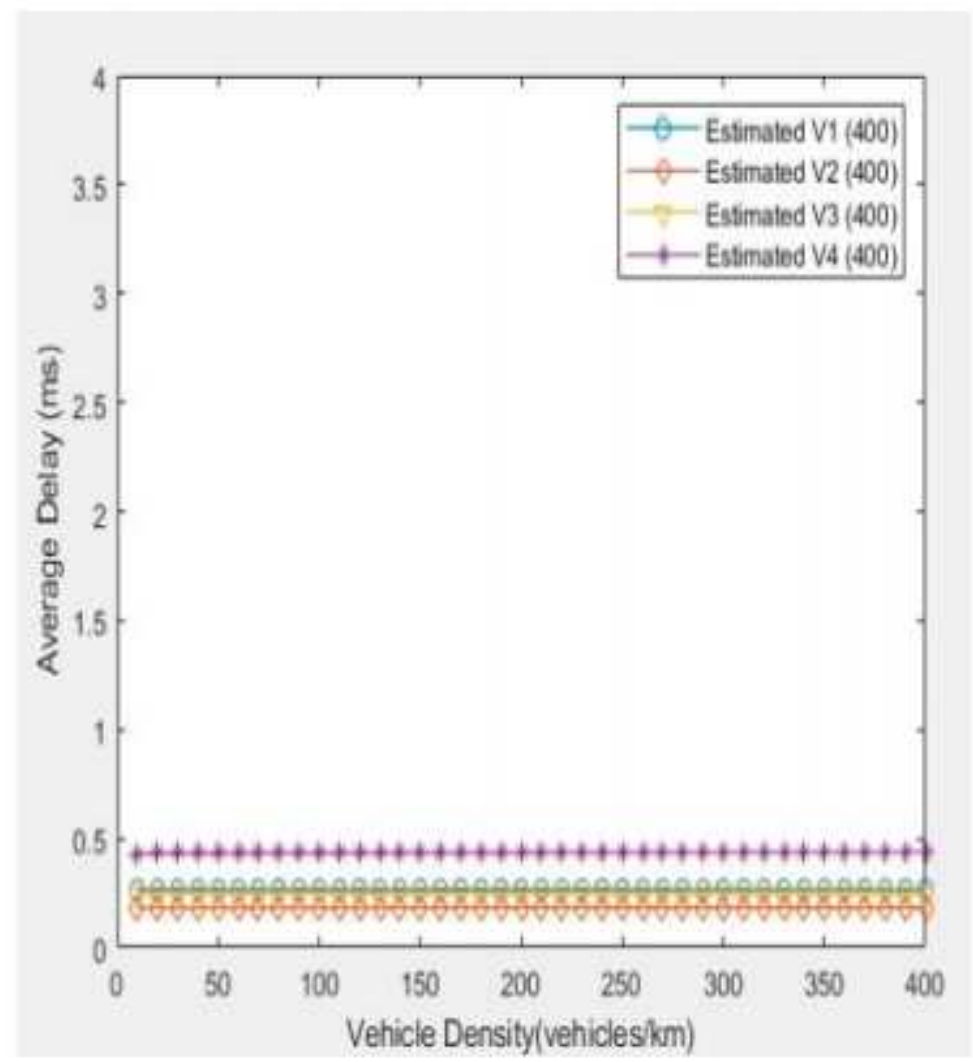

\section{Figure 4}

performance analysis of Average delay (ms)

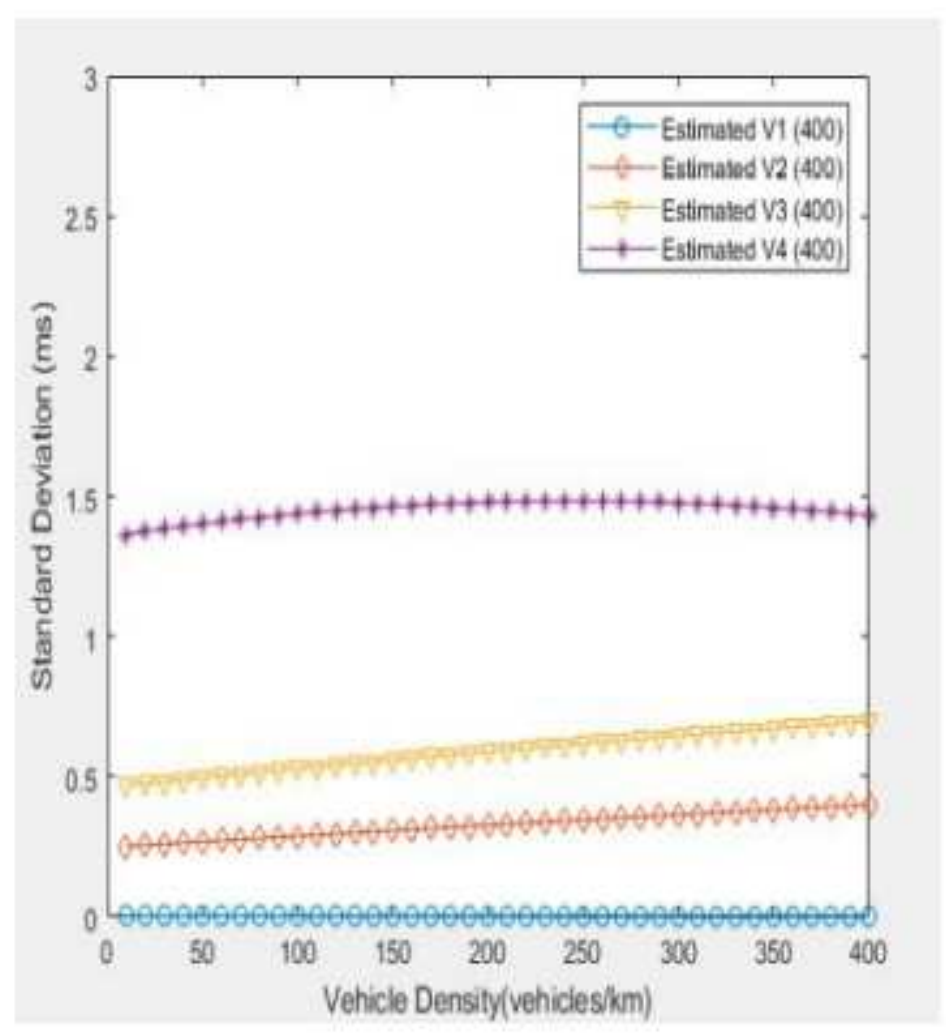


Figure 5

performance analysis of standard deviation Vs vehicle density

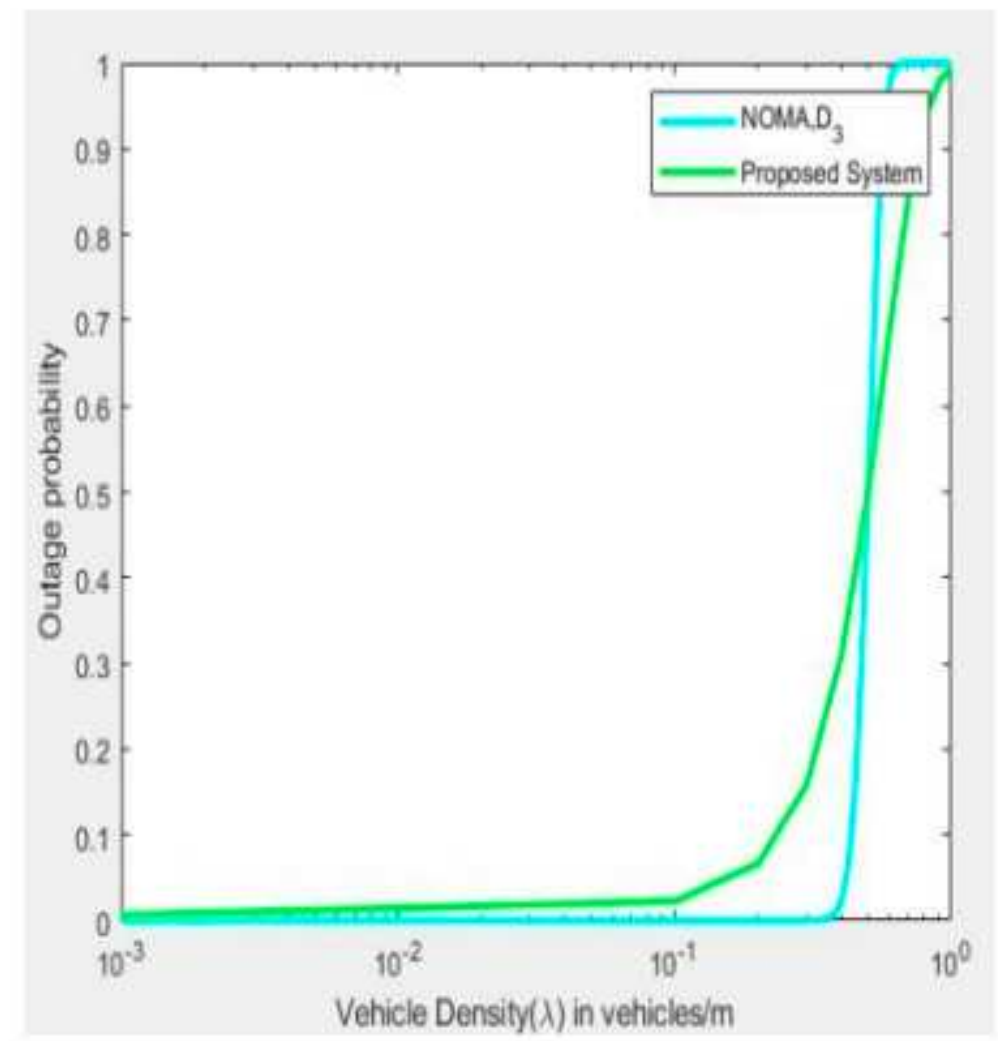

Figure 6

performance analysis of outage probability vs vehicle density

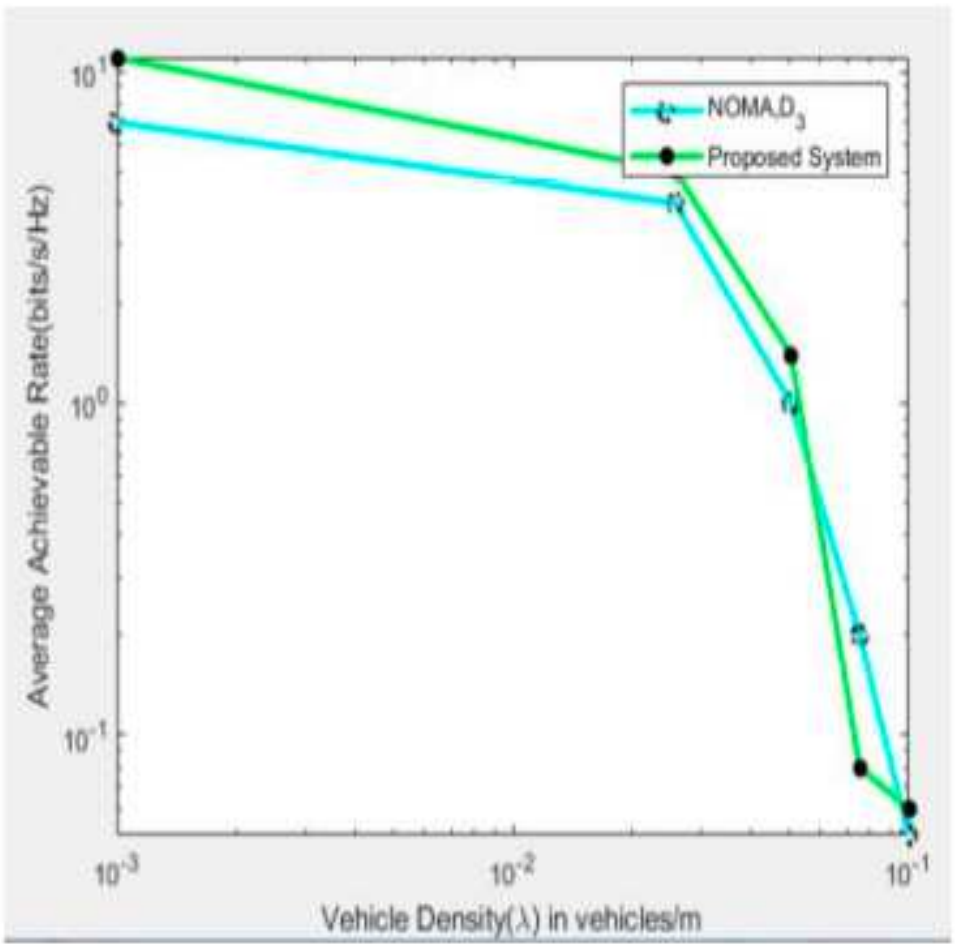


Figure 7

performance analysis of Average achievable rate

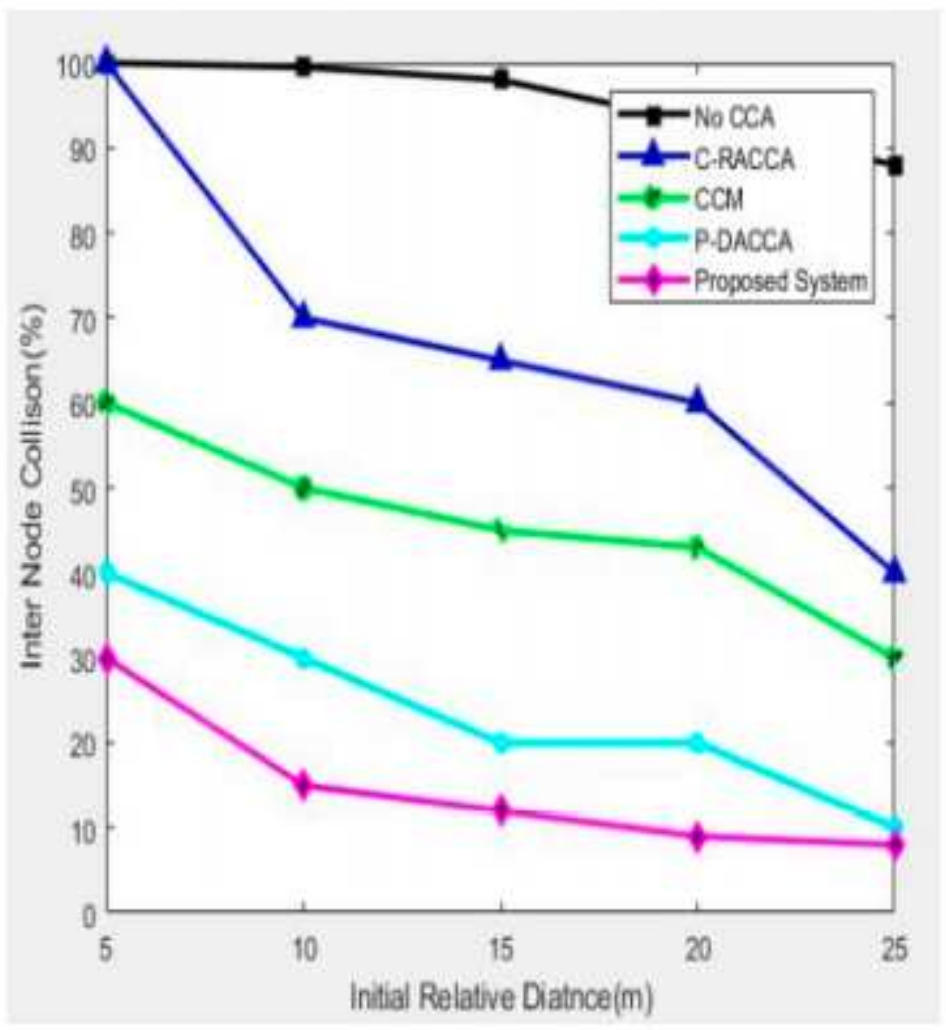

Figure 8

comparative analysis of Inter node collision vs initial relative distance 


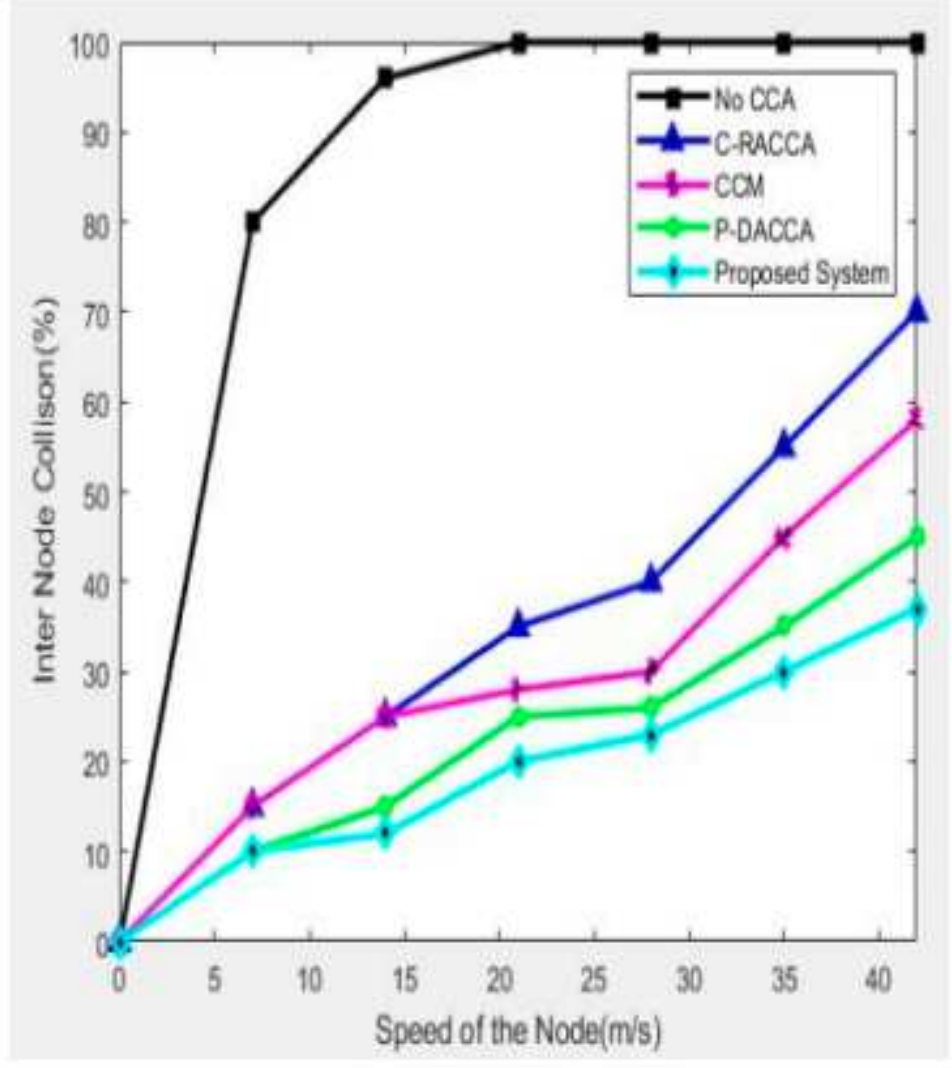

\section{Figure 9}

comparative analysis of Inter node collision Vs speed of the node

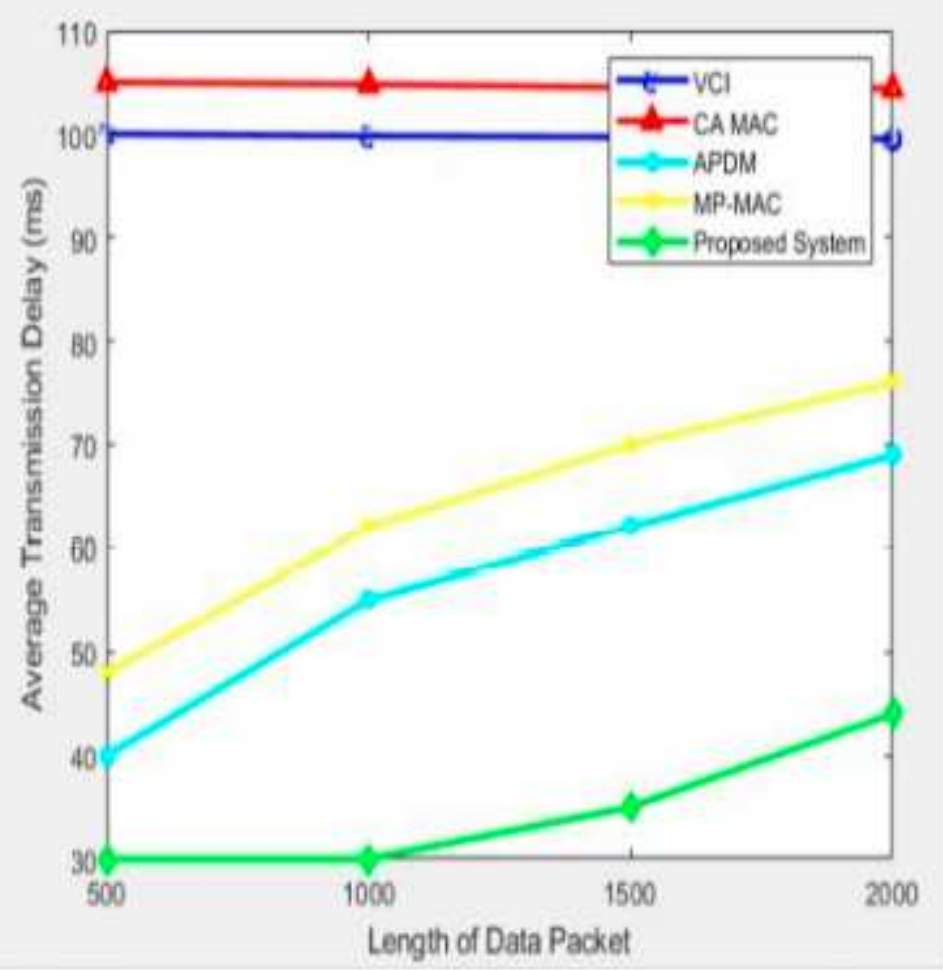

Figure 10 
comparative analysis of Average transmission delay (ms)

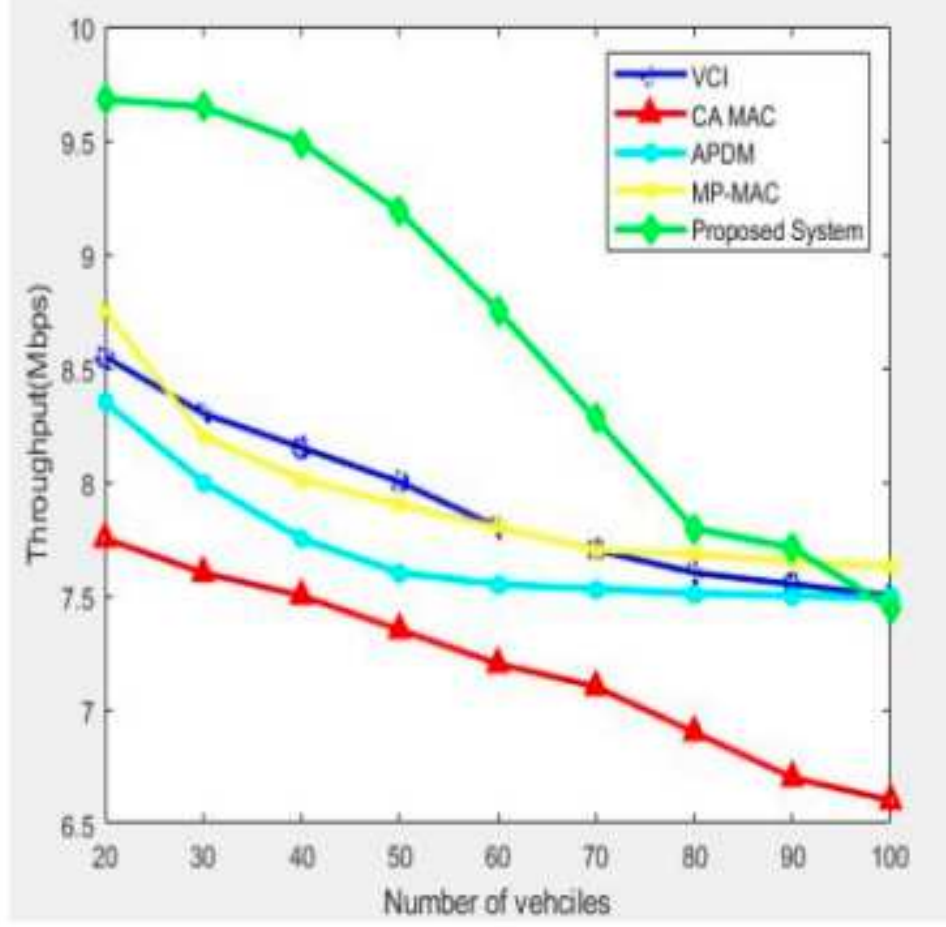

Figure 11

Throughput analysis 